\title{
COVID-19 and Gynecological Cancers: A Summary of International Recommendations
}

\author{
Nabil Ismaili ${ }^{1,2}$ (D) Sanaa Elmajjaoui ${ }^{3}$ \\ Accepted: 20 August 2020 / Published online: 31 August 2020 \\ (C) Springer Nature Switzerland AG 2020
}

\begin{abstract}
Morocco and the rest of the world are experiencing a pandemic of a new coronavirus known as COVID-19 or SARS-CoV-2 (severe acute respiratory syndrome coronavirus 2). On August 24, 2020, the spread of the virus in Morocco had caused more than 52,000 cases and 880 deaths. Cancer patients are more susceptible to develop an infection than people without cancer because of their immunosuppression caused by the disease and treatments (surgery and chemotherapy). Therefore, these patients are at higher risk of infection with COVID-19 and a much higher risk of developing more serious forms. Given this epidemiological context, the establishment of guidelines for patients with gynecological cancers, requiring multidisciplinary management during the global COVID-19 pandemic, is crucial to limit their infection while maintaining their chances for a cure. In this paper, we summarize the international COVID-19 recommendations on the prioritization of surgical cases, the perioperative protective measures, the precautions to be taken in the brachytherapy unit, the COVID-19 screening, and finally the therapeutic indications of gynecological cancers by tumor location.
\end{abstract}

Keywords COVID-19 $\cdot$ Gynecologic cancers $\cdot$ Surgery $\cdot$ Chemotherapy $\cdot$ Radiotherapy $\cdot$ Prioritization $\cdot$ Recommendations

\section{Introduction}

Morocco and the rest of the world are experiencing a pandemic of a new coronavirus known as COVID-19 or SARS-CoV2 (severe acute respiratory syndrome coronavirus 2) [1]. On August 22, 2020, the spread of the virus in Morocco had caused more than 52,000 cases and 880 deaths.

Cancer patients are more susceptible to develop an infection than people without cancer because of their immunosuppression caused by the disease and treatments, such as

This article is part of the Topical Collection on COVID-19

Nabil Ismaili

ismailinabil@yahoo.fr; nismaili@um6ss.ma

Sanaa Elmajjaoui

drsanaamajjaoui@hotmail.com

1 Faculty of Medicine, Mohammed VI University of Health Sciences (UM6SS), Casablanca, Morocco

2 Department of Medical Oncology, Cheick Khalifa International University Hospital, Casablanca, Morocco

3 Department of Radiotherapy, National Institute of Oncology, Mohammed V University, Rabat, Morocco chemotherapy and/or surgery. Therefore, these patients are at higher risk of infection with COVID-19 and a much higher risk of developing more serious forms [1].

Given this epidemiological context, it is important to establish recommendations that may still offer these patients effective treatment while at the same time limit their exposure and infection with the virus.

In fact, a recent Turkish study of 200 patients who received surgery for gynecological cancers during the COVID-19 pandemic showed that gynecological cancer surgery can be performed safely when appropriate measures to protect patients and staff from the exposure to COVID-19 are taken preoperatively, intraoperatively, and postoperatively. Only $1 \%$ of cases developed symptoms of COVID-19 after surgery, and no member of the surgical team developed symptoms related to COVID-19 disease [2].

Two precautions seem to be important for our patients $[3,4]$ :

1) Limiting the consequences of very high-risk situations such as surgery and chemotherapy;

2) Limiting exposure of patients to treatment stations.

To protect patients and healthcare staff form COVID-19 contamination, screening of COVID-19 infection in cancer patients should be recommended before initial management. 
Screening should be based on clinical evaluation of symptoms suggestive on COVID-19 infection, systematic temperature taking at the entrance to cancer units, and, on PCR test, possibly coupled with a chest CT scan, depending on availability, in addition to blood tests to look for eosinopenia, lymphopenia, and an increase in inflammatory parameters.

Teleconsultation is an interesting monitoring tool that ensures continuity of care for cancer patients and thus reduces the unnecessary physical travel to care facilities, by selecting those who really need it.

In this paper, we sought to report useful recommendations to reduce the impact of this pandemic in patients with gynecologic cancers.

\section{Recommendations to Reduce the Spread of the Virus in Hospitals}

To limit the spread of the COVID-19 virus, the Center for Disease Control (CDC) (https://www.cdc.gov/coronavirus/ 2019-ncov) recommends:

- Improving social distancing measures (both inside and outside hospitals)

- Constant using of a face mask outside the home

- Frequent handwashing

- Limiting non-essential activities

- Limitation/eviction of visitors for at least 2 weeks before and 2 to 4 weeks after surgery

- Using teleconsultations for the follow-up and establishment of medical prescriptions

- Using virtual technologies

It is important to note that these measures may have a psychological impact on patients due to the absence of an accompanying person to supporting and helping them with decision-making [5].

For the medical staff the CDC (https://www.cdc.gov/ coronavirus/2019-ncov) recommends:

- Systematic wearing of a face mask at all times in the work

- Meticulous hand hygiene

- Respecting for an interpersonal distance (6 ft)

- Eye protection

Face masks can provide source control (protecting others from respiratory secretions from the mask wearer) and protection (protecting the wearer from secretions from others), but not all masks play both roles. For example, surgical masks provide source control with limited or no wearer protection, while N95 (or FFP2 mask) respirator masks provide source control and wearer protection. N95 respirators are also recommended for the care of COVID19 positive/suspected patients [5].

\section{Pretherapeutic Screening for COVID-19}

Access to rapid and reliable tests to detect the presence of an active infection by the virus has been a major challenge during this pandemic due to the limited capacities of testing. The CDC (https://www.cdc.gov/coronavirus/2019-ncov) and many medical institutions rapidly developed PCR tests in nasopharyngeal or oropharyngeal swabs.

\section{Clinical Screening for COVID-19}

Clinical screening is based on the evaluation of symptoms of a viral infection (fever, dry cough, tiredness, etc.), systematic temperature taking at the entrance to cancer units, and research of potential contact with high-risk individuals, including those in quarantine. All patients should undergo clinical screening by teleconsultation prior to visit, before the entrance to the cancer center, and immediately prior to surgery, etc. [5].

\section{COVID-19 PCR Testing}

Diagnostic testing involves identifying specific genes for SARS-CoV-2 (core or envelope) by real-time RT-PCR, with most results expected within 12 to $48 \mathrm{~h}$. Theoretically, RTPCR assays for SARS-CoV-2 is very sensitive to detect the presence of viral RNA. However, the limit of detection differs for different respiratory tract sources. The performance of RTPCR from nasopharyngeal secretions varies depending on the time since symptom onset or exposure to COVID-19 virus, the false-negative rate ranged from 20 to $100 \%$. Routine preoperative COVID-19 testing is recommended due to the high prevalence of asymptomatic cases. A single negative test cannot exclude the wearing of N95 masks, when available, during AGP (aerosol-generating procedures). In surgical emergencies, surgery should be performed on the assumption that the patient is positive if test results are not available [5].

\section{Computed Tomography Scan}

Preoperative screening by chest computed tomography (CT) may be relevant in patients at risk. Indeed, nearly $19 \%$ of COVID-19 pneumonia occurs in asymptomatic patients. In settings where RT-PCR testing is not available and in areas of high prevalence of COVID-19, chest CT may be the only option as a preoperative test. However, the CDC (https:// www.cdc.gov/coronavirus/2019-ncov) and the American College of Radiology do not recommend performing chest $\mathrm{CT}$ as a test for COVID-19 but reserve it for diagnostic purposes in symptomatic patients [5]. 


\section{Precautions in Surgery Unit}

\section{Triage and Surgical Prioritization in Gynecological Oncology}

The American College of Surgeons (ACS) and other international societies have established recommendations to protect patients and medical staff from COVID-19 contamination and to preserve hospital resources. Patients with gynecological cancers often require surgery which is particularly disrupted during the COVID-19 pandemic. Consequently, accurate triage of urgent vs elective surgical cases is crucial in order to preserve the availability of medical resources to face a growing number of COVID-19-positive patients. In addition, hospitals are an important vector for the virus spread therefore guidelines should be strategic to ensure the safety of patients and staff $[2,5]$.

Most cancer-related interventions, including those for gynecologic cancers, are given a high priority level (semiurgent) $[2,5]$.

The surgical prioritization is determined according to:

- Local resources

- Prevalence of COVID-19 disease

- Availability of masks

- Patient's comorbidities and tumor characteristics

- Length of stay in hospital and intensive care unit

- Patient's need for blood products

- Expected impact of the surgery delays

The highest priority should be given to the surgical treatment of high-risk cancers, especially those candidates for minimally invasive surgery (such as robotic surgery) and short hospital stay, in addition to potentially curative procedures for early-stage cancers. In addition, women with advanced ovarian and uterine cancers, requiring cytoreductive interval surgery after neoadjuvant chemotherapy should be given priority when possible. However, in the restricted surgical areas of high COVID-19 prevalence, cytoreduction (primary/interval) should be postponed, and chemotherapy started (or continued up to 6 cycles) to prevent exposure of immunocompromised patients to the virus and to conserve hospital resources [5].

According to the American Society for Colposcopy and Cervical Pathology (ASCCP), surgery for high-grade dysplasia may be delayed for up to 3 months and dysplasia of lower grade surgery can be delayed for 6 to 12 months. In contrast, patients with invasive disease should be treated within 1 month [5].

\section{Preoperative Precautionary Measures}

During surgery, the most dangerous time in a COVID-19positive or unknown patient is endotracheal intubation and extubation considered as aerosol-generating procedures (AGP). AGPs are more likely to generate higher concentrations of infectious respiratory aerosols than coughing, sneezing, speaking, or breathing. Therefore, enhanced security measures are necessary [5].

The following recommendations allow a fast and safe intubation/extubation process for all operating room staff [5]:

- Only necessary personnel should be in the operating room during the intubation/extubation process. The rest of the surgical team should stay out of the room, and the time of re-entry depends on the type of mask they wear and the level of air renewal in the room. In a standard operating room, $99 \%$ of airborne pathogens are cleared from the room within $18 \mathrm{~min}$, after which time staff can re-enter safely with surgical masks and eye protection. The surgeon and operating room staff may also wear an N95 respirator with goggles if they cannot leave the room (patient not masked).

- Rapid sequence intubation and video laryngoscopy reduce the risk of aerosolization and promote accuracy and increase the distance between the intubator and the patient.

- All surgeons should wear the usual cap, gowns, protective shoes, and double gloves. N95 masks and eye protection are recommended for AGP procedures; a surgical mask with eye protection can be used for non-AGP procedures.

\section{The Role of Robotic Surgery}

Robotic surgery has major advantages over laparoscopic surgery. Robotic surgery can be performed safely with pressures low than $10 \mathrm{mmHg}$. This lower pressure requirement probably reduces the risk of transmission of viral particles to the medical staff. Another advantage is the reduced number of healthcare professionals in an operating room and the distance between team members. In conventional laparoscopic surgery, the surgical team traditionally includes the surgeon, an assistant, a technician, and a nurse. These members are very close to each other. With robotic surgery, the surgeon's console is separated from the robot, providing more space between team members. Additionally, robotic surgery may require fewer assistants, which means less overall exposure [6].

\section{Precautions in Brachytherapy Unit [7]}

During the COVID-19 pandemic, precautions should be done in order to reduce the spread of the virus in brachytherapy unit:

- Management of cancer patients should be done in "COVID-19-negative" units 
- Establishment of circuit dedicated to patients suspected with COVID-19 infection

- Establishment of priority lists to reduce the number of patients and limit the risk of exposure with other patients and staff

- Limit the number of patients waiting in the waiting room

- Monitor all patients at admission to brachytherapy unit, for symptoms suggestive of COVID-19 infection: fever, cough, or dyspnea, and take their temperature

- Prohibit access to accompanying persons to the service whenever possible

- Mandatory wearing of protective masks by patients and staff

- Reducing the number of hospitalizations in the brachytherapy unit (one patient per room) to maintain a sufficient distance between patients

There are no major changes in brachytherapy procedures. Patients without COVID-19 symptoms, who have already started their intracavitary brachytherapy (ICBT), should complete their schedule without delay. Under normal conditions, all ICBT applications should be done using spinal anesthesia and ultrasound guidance, however, in the case of lack of anesthesiologist and their staff, consider pudendal nerve block or local anesthesia by paracervical block or by the use of medical hypnosis technique.

For patients with cervical cancers, to minimize the risk of contamination, prefer the schemes with a reduced number of fractions: $3 \times 8$ Gy or $4 \times 7$ Gy by using an HDR ICBT schedule of 7 Gy per fraction for 4 fractions in 2 insertions 1 week apart. For patients over 70 years of age or with significant comorbidities who have small tumors or responding well to external beam radiotherapy (EBRT), a more shortened schedule of 9 Gy per fraction in 2 fractions at 1 week apart may be considered.

In stage I, low-risk endometrial cancer, it is recommended to postpone brachytherapy up to 12 weeks. For patients with significant comorbidities, exposing them to the risk of severe complications of COVID-19 infection, it may be judicious to delay brachytherapy for 6 months. Patients, who should start vaginal vault brachytherapy (VVB), should consider the schedule of 7 Gy for 3 fractions, to limit the number of patient displacement. An interval spacing of 14 days between the fractions is also acceptable. However, for high-risk patients who received adjuvant external RT, omitting BVV may be reasonable.

For stage II endometrial cancers, adjuvant VVB is delivered exclusively (if invasion $<50 \%$ of the myometrium, G1 and 2) or after EBRT (if invasion $>50 \%$ of the myometrium, G3). In both cases, consider to postpone brachytherapy by 1 to 2 months. In the case of a COVID-19-positive patient, postpone treatment for at least 24 days [7].
Multidisciplinary Management by Tumor Site

\section{Cervical Cancer}

For low-grade cervical dysplasia, the diagnostic evaluation may be postponed for 6 to 12 months. However, in the case of high-grade dysplasia (CIN3 or CIS), the diagnostic evaluation should be done within 3 months [4, 5].

For patients with early-stage cervical cancer (FIGO IA, IB1, IB2, and IIa stages), if surgery is still allowed in the institution, consider standard treatment with surgery. However, when access to surgery is limited, the following procedures may be considered $[3-6,8]$ :

- For limited disease, consider to postpone surgical treatment (trachelectomy or radical hysterectomy) for a period of 8 weeks or until the crisis resolves;

- For microscopic disease (stage IA) or low-risk disease $(<$ $2 \mathrm{~cm}$, low-risk histology excluding adenocarcinoma and small cell carcinoma), consider conization or a simple trachelectomy + sentinel node mapping (if available) or pelvic lymphadenectomy;

- For large tumor $>2 \mathrm{~cm}$ (stage IB2 or IIa), surgical staging is more accurate than clinical and radiological staging and should be discussed on a case by case basis. The primary treatment is either surgery or radiotherapy/brachytherapy. Radical hysterectomy with bilateral pelvic lymph node dissection \pm para-aortic lymph node dissection is the preferred surgical option. Radiotherapy/brachytherapy should be considered in the case of limited access to surgery or for patients who are not eligible for hysterectomy [9].

For locally advanced stages of the disease (FIGO stages IB3, IB2-IVA), concurrent chemoradiotherapy followed by brachytherapy is recommended and should be started on time without delay $[7,8]$. Consider hypo-fractionated radiotherapy to reduce the frequency of patient exposure to treatment stations [4].

Patient with metastatic cervical cancer should be considered for first-line chemotherapy based on paclitaxelcarboplatin \pm bevacizumab (prefer carboplatin to cisplatin) [10]. However, second-line treatment has a low benefit and limited priority.

\section{Endometrial Cancer}

For early-stage disease (IA and IB), surgery is the primary treatment $[11,12]$. Minimally invasive laparoscopic surgery/ robotic surgery results in lower perioperative complication and should be the preferred option if available [611]. Useful preoperative prognostic factors to guide treatment strategy are age, lympho-vascular invasion, tumor size, depth of uterine 
invasion, and lymph node involvement [11]. For low-risk cancers (stage IA, grade 1 and 2), consider total hysterectomy and bilateral salpingo-oophorectomy (TH/BSO) with sentinel lymph node procedure (if available) or lymph node dissection. For intermediate risks (IB, grade 1 and 2), radical surgery plus vaginal brachytherapy is the preferred strategy $[4,7,11]$. For elderly women and for patients with comorbidities, initial systemic therapy with hormone therapy (for estrogen and progesterone receptor positive), and postpone surgery until the crisis resolves $[4,11]$. For high-risk cancers (IB grade 3 or high-risk histology type 2), the recommended surgical procedure includes TH/BSO with surgical staging and lymph node dissection in a single-incision surgery [11]. Adjuvant treatment with radiotherapy without brachytherapy is recommended and should be started within 3 months [7, 13, 14]. Systemic adjuvant therapy using carboplatin AUC5 plus paclitaxel $175 \mathrm{mg} /$ $\mathrm{m}^{2}$ for 4 cycles is to be considered in high-risk disease. Consider granulocyte colony-stimulating factors (GCSF) to limit the risk of febrile neutropenia. In stage II endometrial cancer, proceed with radical surgery and consider adjuvant therapy for patients with high-risk factors (same as stage I). For locally advanced stages (III and IVA), surgical treatment is the treatment of choice $[3,4,11]$. Adjuvant treatment with radiotherapy \pm vaginal brachytherapy is recommended. In the adjuvant setting, chemotherapy with carboplatin plus paclitaxel (plus GCSF) is the preferred regimen for 6 cycles $[15,16]$. For patients who are not suited for surgery, radiotherapy/ brachytherapy is an effective treatment and may be considered with or without chemotherapy [17]. For metastatic disease (FIGO IVB stage), chemotherapy based on carboplatin/ paclitaxel plus GCSF is recommended. Elderly patients or pauci-symptomatic patients with low-grade histology favor mono-chemotherapy (paclitaxel, doxorubicin, carboplatin) or hormone therapy (for hormone receptor-positive cancers) [3, 4].

\section{Ovarian Cancer}

Early-stage ovarian cancer proceeds with oophorectomy on the suspect mass; then, the decision to complete surgical staging is based on final histological analysis [3,4]. Consider adjuvant chemotherapy with carboplatin-paclitaxel (4 cycles) for patients with high-grade serous and endometrial ovarian carcinoma. For early-stage non-serous, non-endometrioid, or low-grade histological cancers, the benefit of chemotherapy is controversial and surveillance is the preferred option. For stages IC and II (with high-risk factors), consider adjuvant chemotherapy with paclitaxel-carboplatin plus GCSF for 4 cycles [18]. For patients with advanced stage (stages III/IV) high-grade epithelial (serous or endometrioid) ovarian cancer, the standard treatment is based on primary debulking surgery and adjuvant chemotherapy (protocol based on the result of the surgery). However, during the COVID-19 pandemic, neoadjuvant chemotherapy (NAC) strategy is the preferred option even in patients who are candidates for primary surgery because of the occupancy of intensive care unit beds by COVID19 patients [5]. In addition, randomized trials have reported a significant reduction in operative time and complications in favor of NAC. Carboplatin AUC 5/paclitaxel $175 \mathrm{mg} / \mathrm{m}^{2} \mathrm{ev}-$ ery 3 weeks for 6 cycles is the recommended regimen, following neoadjuvant treatment, consideration of maximum debulking surgery with total hysterectomy $(\mathrm{TH})$, bilateral salpingo-oophorectomy (BSO), omentectomy, and complete resection of all visible disease. In the case of R0 surgery first, adjuvant treatment based on carboplatin/paclitaxel for 6 cycles is recommended without maintenance chemotherapy $[18,19]$. For patients with high-risk factors ( $\mathrm{R} 1$ and $\mathrm{R} 2$ resection, and stage IV disease), maintenance treatment with bevacizumab is recommended [20]. For patients with a germ line or somatic BRCA1/2 mutation, maintenance therapy with PARP inhibitor is the preferred option (if available) [4]. For patients receiving neoadjuvant chemotherapy, consideration of extending treatment for six cycles until the crisis resolves and postpone surgery at a later date, due to the unavailability of ICU beds [3-521]. For patients not eligible for surgery, chemotherapy with carboplatin/paclitaxel and bevacizumab for 6 cycles followed by maintenance bevacizumab is recommended. For patients with BRCA mutation, consider the early start of maintenance therapy with PARP inhibitors after 4 cycles of chemotherapy $[18,19]$. After disease progression, chemotherapy may be indicated for symptomatic patients [3, 4].

\section{Vulvar Cancer}

For early-stage vulvar cancer ( $\mathrm{T} 1$ or $\mathrm{T} 2$ ), primary surgery is the treatment of choice [21]. Inguino-femoral lymph node dissection is recommended for T1 or T2 stages with a depth of invasion $>1 \mathrm{~mm}$. Consider adjuvant radiotherapy for high-risk disease with adverse prognostic factors in the pathology report (lymph node involvement, positive vascular emboli, marginal $<8 \mathrm{~mm}$ or positive tumor margins, tumor size $>4 \mathrm{~cm}$, and or depth of invasion) [22]. When surgical management would require very heavy surgery, consider concurrent chemoradiotherapy. For elderly women with early-stage disease, postpone treatment by $4-8$ weeks. For locally advanced vulvar cancer (T2-T3), standard treatment is based on concurrent chemoradiotherapy with cisplatin or 5-FU/cisplatin[21]. For metastatic disease, consider radiotherapy for symptoms palliation and local control [22]. Chemotherapy and supportive care are two alternatives in the context of the COVID-19 pandemic [3].

\section{Vaginal Cancer}

For early-stage I squamous cell carcinoma of the vagina, if $<5 \mathrm{~mm}$ of invasion, exclusive brachytherapy is the treatment of choice. In patients with significant comorbidities (old age, chronic respiratory pathology or cardiac, immunosuppression 
...), consider to postpone brachytherapy by 1 to 2 months. If invasion $>5 \mathrm{~mm}$, consider radiotherapy followed by brachytherapy.

For patients with stage I vaginal adenocarcinoma, consider radical vaginectomy and hysterectomy with lymph node dissection. Adjuvant RT should be considered in the case of close or positive surgical margins[23].

For patients with advanced stage vagina cancer (II, III, and Iva), radio-chemotherapy and brachytherapy are the treatment of choice [3]. For stage IVb vaginal cancer, palliative chemotherapy based on 5fluorouracil-carboplatin is the preferred option [7, 23].

\section{Management of COVID-19-Positive Patients}

Recognizing that the morbidity and mortality from postoperative COVID-19 pneumonia is significant, surgery should be delayed in COVID-19-positive patients until they are asymptomatic for more than 3 weeks; some teams recommend a negative control test by RT-PCR $[1,5]$.

The largest series $(n=121)$ analyzing patients with gynecological cancer and COVID-19 infection is that of the New York City, an area highly exposed to the coronavirus. Overall mortality among patients infected with COVID-19 and having gynecologic cancer was $14 \%$, while mortality among hospitalized patients was $25.8 \%$. These results show that the mortality rate among patients hospitalized in this series is lower compared with the rate reported in the general population of the New York region which is $34.3 \%$. This suggests that patients with gynecologic cancer and COVID-19 infection do not have a higher risk of COVID-19-related mortality compared with the general population [24].

A dedicated circuit should be implemented for COVID-19positive patients whose management cannot be postponed. During urgent surgery for COVID-19-positive patients, patient and staff safety must be the priority. Each center should consider assigning a dedicated team of surgeons to provide surgical care for COVID-19 patients. Professional at high risk of developing severe forms of COVID-19 should avoid participating in the surgical care of COVID-19-positive patients. According to the CDC (https://www.cdc.gov/coronavirus/ 2019-ncov), these persons are those aged $>65$ or those with chronic lung disease, moderate/severe asthma, severe heart disease, immunosuppressive disease or therapy, cancer, etc. It is also desirable to limit the exposure of pregnant women to COVID-19 patients[1, 5].

\section{Follow-up}

- For patients who traveled for long distances for their management, consider treatment and follow-up with local oncologists, to avoid travel and to decrease the risk of exposure and infection.

- Use telecommunications for toxicity evaluation and follow-up.

- Postpone follow-up visits for three months.

- Follow-up of endometrial and/or cervical cancers using alternate clinical examination and teleconsultation.

\section{Summary of Recommendations by Priority}

\section{Recommendations for Chemotherapy}

Very high priority: curative chemotherapy with high benefit $(>50 \%)$ :

Chemotherapy for trophoblastic and germ cell tumors. Concurrent chemoradiation in cervical, vaginal, and vulvar cancers.

High priority: curative treatment with intermediate benefit (15 to $50 \%$ ) compared with local treatment alone:

Chemotherapy in high-grade epithelial ovarian cancer.

Medium priority: curative treatment with low benefit (10$15 \%)$ :

High-grade ovarian cancer during the first platinumsensitive relapse.

High-grade advanced endometrial cancer.

Low priority: palliative treatment with high benefit:

Palliative chemotherapy in the treatment of metastatic cervical, vaginal, vulvar, and endometrial.

Very low priority: palliative treatment with low benefit

Chemotherapy in platinum-resistant ovarian cancer.

\section{Recommendations for Radiotherapy}

General recommendations:

- Access to surgery may be limited in some institutions; therefore, curative radiotherapy may be recommended in certain cases of localized cancers (cervical, vulvar, and vaginal cancers).

- Prioritize access to chemotherapy for concurrent chemoradiation. 
- Check the availability of anesthesia specialists for brachytherapy.

- Favor hypo-fractionated radiotherapy if possible.

Priority levels of radiotherapy:

- High priority: cancers treated with chemoradiotherapy and brachytherapy, palliative radiotherapy for emergencies:

- Radical radiotherapy plus brachytherapy for cervical cancer.

- Radiotherapy for vulvar and vaginal cancers.

- Palliative radiotherapy in the case of spinal cord compression,

- Hemostatic radiotherapy.

- Medium priority: postoperative radiotherapy:

- Adjuvant radiation therapy for residual disease, positive margins, or lymph node involvement in cervical, vaginal, vulvar, and endometrial cancers.

- Low priority:

Palliative radiotherapy for symptom control: palliative radiotherapy for metastases and symptomatic pelvic masses.

Authors' Contributions All authors contributed to the conception and design, drafting, and critical revision of this manuscript. All authors have given final approval of this version to be published, and all authors accept responsibility for its contents.

\section{Compliance with Ethical Standards}

Conflict of Interest The authors declare that they have no conflict of interest.

Ethical Approval This article does not contain any studies with human participants performed by any of the authors.

\section{References}

1. Ismaili N. COVID-19 Recommendations for patients with cancer: the post-COVID-19 era. SN Compr Clin Med. 2020. https://doi. org/10.1007/s42399-020-00425-7.

2. Dursun P, Dervisoglu H, Daggez M, et al. Performing gynecologic cancer surgery during the COVID-19 pandemic in Turkey: A multicenter retrospective observational study. Int J Gynaecol Obstet. 2020. https://doi.org/10.1002/ijgo.13296.

3. Akladios C, Azais H, Ballester M, et al. Prise en charge chirurgicale des cancers gynécologiques en période de pandémie COVID-19Recommandations du Groupe FRANCOGYN pour le CNGOF. Gynecol Obstet Fertil Senol. 2020;48(5):444-47. https://doi.org/ 10.1016/j.gofs.2020.03.017.

4. Ramirez PT, Chiva L, Eriksson AGZ, et al. COVID-19 global pandemic: options for management of gynecologic cancers. Int $\mathrm{J}$ Gynecol Cancer. 2020;30(5):561-63. https://doi.org/10.1136/ijgc2020-001419.

5. Fader AN, Huh WK, Kesterson J, Pothuri B, Wethington S, Wright JD, et al. When to operate, hesitate and reintegrate: Society of Gynecologic Oncology Surgical Considerations during the COVID-19 pandemic. Gynecol Oncol. 2020;158(2):236-43.

6. Moawad GN, Rahman S, Martino MA, Klebanoff JS. Robotic surgery during the COVID pandemic: why now and why for the future. J Robot Surg. 2020:1-4. https://doi.org/10.1007/s11701-02001120-4.

7. ElMajjaoui S, Ismaili N, Benjaafar N. COVID-19, brachytherapy, and gynecologic cancers: a Moroccan experience. SN Compr Clin Med. 2020:1-4. https://doi.org/10.1007/s42399-020-00402-0.

8. Koh WJ, Abu-Rustum NR, Bean S, et al. Cervical cancer, version 3.2019, NCCN Clinical Practice Guidelines in Oncology. J Natl Compr Cancer Netw. 2019;17(1):64-84. https://doi.org/10.6004/ jncen.2019.0001.

9. Landoni F, Maneo A, Colombo A, Placa F, Milani R, Perego $\mathrm{P}$, et al. Randomised study of radical surgery versus radiotherapy for stage Ib-IIa cervical cancer. Lancet. 1997;350(9077): $535-40$

10. Tewari KS, Sill MW, Long HJ 3rd, Penson RT, Huang H, Ramondetta LM, et al. Improved survival with bevacizumab in advanced cervical cancer. N Engl J Med. 2014;370(8): 734-43.

11. Koh WJ, Abu-Rustum NR, Bean S, et al. Uterine neoplasms, version 1.2018, NCCN Clinical Practice Guidelines in Oncology. J Natl Compr Cancer Netw. 2018;16(2):170-99.

12. American College of Obstetricians and Gynecologists. ACOG practice bulletin, clinical management guidelines for obstetrician-gynecologists, number 65, August 2005: management of endometrial cancer. Obstet Gynecol. 2005 Aug; 106(2):413-25.

13. Scholten AN, van Putten WLJ, Beerman H, et al. Postoperative radiotherapy for stage 1 endometrial carcinoma: long-term outcome of the randomized PORTEC trial with central pathology review. Int J Radiat Oncol Biol Phys. 2005;63:834-8.

14. Keys HM, Roberts JA, Brunetto VL, Zaino RJ, Spirtos NM, Bloss JD, et al. A phase III trial of surgery with or without adjunctive external pelvic radiation therapy in intermediate risk endometrial adenocarcinoma: a Gynecologic Oncology Group study. Gynecol Oncol. 2004;92(3):744-51.

15. Jutzi L, Hoskins P, Lim P, et al. The importance of adjuvant chemotherapy and pelvic radiotherapy in high-risk early stage endometrial carcinoma. Gynecol Oncol. 2013;581-585(207): 131.

16. de Boer SM, Powell ME, Mileshkin L, et al. Toxicity and quality of life after adjuvant chemoradiotherapy versus radiotherapy alone for women with high-risk endometrial cancer (PORTEC-3): an openlabel, multicentre, randomised, phase 3 trial. Lancet Oncol. 2016;17:1114-26.

17. van der Steen-Banasik E, Christiaens M, Shash E, Coens C, Casado A, Herrera FG. Ottevanger PB; European Organisation for Research and Treatment of Cancer, Gynaecological Cancer Group (EORTC-GCG). Systemic review: radiation therapy alone in medical non-operable endometrial carcinoma. Eur J Cancer. 2016;65: 172-81.

18. Morgan RJ Jr, Armstrong DK, Alvarez RD, et al. Ovarian cancer, version 1.2016, NCCN Clinical Practice Guidelines in Oncology. J Natl Compr Cancer Netw. 2016;14(9):1134-63.

19. Armstrong DK, Alvarez RD, Bakkum-Gamez JN, et al. NCCN Guidelines Insights: ovarian cancer, version 1.2019. J Natl Compr Cancer Netw. 2019;17(8):896-909.

20. Oza AM, Cook AD, Pfisterer J, ICON7 trial investigators, et al. Standard chemotherapy with or without bevacizumab for women with newly diagnosed ovarian cancer (ICON7): overall survival results of a phase 3 randomised trial. Lancet Oncol. 2015 Aug;16(8):928-36.

21. Ismaili N. COVID-19 and gynecological cancers: a Moroccan point-of-view. Radiother Oncol. 2020;148:227-8. 
22. Koh WJ, Greer BE, Abu-Rustum NR, et al. Vulvar cancer, version 1.2017, NCCN Clinical Practice Guidelines in Oncology. J Natl Compr Cancer Netw. 2017 Jan;15(1):92-120.

23. PDQ Adult Treatment Editorial Board. Vaginal Cancer Treatment (PDQ®): Health Professional Version. PDQ Cancer Information Summaries [Internet]. Bethesda (MD): National Cancer Institute (US); 2002-.2020
24. Lara OD, O'Cearbhaill RE, Smith MJ, Sutter ME, Knisely A, McEachron J, et al. COVID-19 outcomes of patients with gynecologic cancer in New York City. Cancer. 2020. https://doi.org/10. $1002 /$ cncr.33084.

Publisher's Note Springer Nature remains neutral with regard to jurisdictional claims in published maps and institutional affiliations. 